\title{
Exploring Sketch Beautification Techniques
}

\author{
Beirong Wang, Jian Sun, Beryl Plimmer \\ University of Auckland \\ Private Bag 92019, Auckland \\ New Zealand. Phone: +64 93033670
}

\author{
\{bwan024|jsun027\}@ec.auckland.ac.nz, beryl@cs.auckland.ac.nz
}

\begin{abstract}
Beautification of vague, imprecise sketchy ink input is an interesting area for exploration, especially with the emergence of pen-based systems, such as the Tablet PC. Fifty percent of the total time spent creating drawings on a computer is on formalisation operations [3], why waste this time when the same result is achievable via recognition and beautification techniques? We examined beautification and its value in supporting the design process by prototyping a design tool incorporating several beautification techniques. The following is a description of the design, construction and evaluation of our grid based design environment.
\end{abstract}

\section{Categories and Subject Descriptors}

H.5.2 [User Interfaces]: Input devices and strategies, I.3.6 [Methodology and Techniques] Interaction techniques

\section{Keywords}

Pen-based computing, ink beautification

\section{INTRODUCTION}

Designers prefer the traditional environments, such as pen and paper, for rendering ideas in the early stages of the creative process [1]. However final diagrams are presented as formal, geometrically accurate renderings. Beautification is the process of translating the hand-drawn sketch to a tidy regular diagram. This takes time, wasting much design effort. Our goals are (a) to create a stylus input design tool for the tablet PC (b) that incorporates a range of beautification techniques and (c) to establish the effectiveness of the different beautification techniques.

\section{RELATED WORK}

Pavlidis and Van Wyk [2] developed a process where constraints are imposed on the original diagram content to formalise the content by both standardising sizes and aligning widgets. Bolz [3] then devised techniques to critique and beautify diagrammatical input via a user-defined knowledge base. Pegasus [6] pioneered interactive beautification, transforming pen strokes into straight line segments taking the geometric properties of design and its inherent ambiguity into account. Arvo and Novins [5] used instantaneous morphing of stylus input to create regular geometric shapes. These investigations into beautification and recognition

Permission to make digital or hard copies of all or part of this work for personal or classroom use is granted without fee provided that copies are not made or distributed for profit or commercial advantage and that copies bear this notice and the full citation on the first page. To copy otherwise, or republish, to post on servers or to redistribute to lists, requires prior specific permission and/or a fee.

CHINZ'05, July 6-8, 2005, Auckland, NZ

Copyright 2005 ACM 1-59593-036-1/04/10 enable raw sketches to become computer-formalised diagrams. However, beautification of sketchy content early on in the design process often hinders the designers' motivation for further exploration [1]. Investigation of appropriate beautification techniques and their effective deployment is the purpose of this work.

\section{DESIGN}

We propose an application which aids design in the Tablet PC paradigm and tests the supportiveness of various beautification techniques in the creative process. This presents two major challenges: constructing an intuitive, stylus-input interface incorporating the standard design application functionalities; and implementing a range of beautification techniques. Additionally, the equally important evaluation of such a tool requires testing for not only usability of the application itself, but also the potential usefulness of each beautification technique.

Input through the stylus simulates traditional renderings of creative ideas using pen and paper, providing users with the freedom to explore early design concepts; while recognition and beautification offer users the options for editing, storing and sharing their ideas as well-defined diagrams [1]. However, certain challenges remain in designing an effective drawing application user interface for the tablet such as parallax errors and the overloaded functionalities of the pen [7].

We recognise that different types of sketch-based applications may require different beautification techniques, which can be deployed at different times [4]. Only when the type and timing of the beautification technique are tailored to the nature of the design imperative can the creative process be effectively enhanced. To determine the most suitable beautification technique and the stage in the drawing process where it can most effectively be deployed, we propose to implement and test six beautification techniques, which lie on a continuum, differing in nature and timing of deployment. Four ink-transformation techniques are proposed: manual, continuous morphing, corner triggered and pen-up triggered (figure 1: a, b, c and d respectively); and two shadowing techniques: formal shadowing and sketch shadowing (figure 1: e and $f$ respectively).

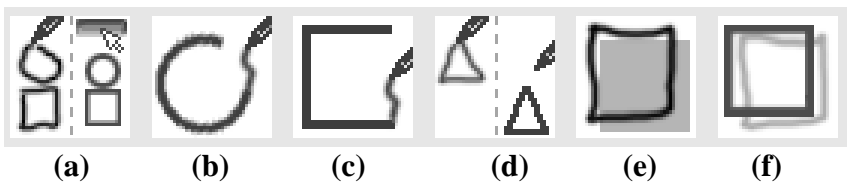

Figure 1. Images illustrating beautification techniques.

On-going transformation of the hand-drawn input into formalised drawings allows users to visualise the beautification process. We work with a visible grid because many diagrams naturally fall onto a grid and, as our goal is to explore beautification, we choose to explicitly guide the user. 
The manual beautification technique gives the user the most control, as beautification and recognition is triggered only when the user clicks a button. Continuous beautification, the other end of the continuum, is when raw sketches are constantly recognised, beautified and transmuted into the most suitable formal shape.

Ink strokes drawn by the user in the corner triggered beautification technique are formalised into computer-based geometric elements when the user passes a grid point or returns to the starting grid point (for the rendering of a circle for example). Pen-up triggered is similar, except the recognition and beautification is performed when the user lifts the pen off the surface.

The formal shadowing technique leaves the rough hand-drawn shapes on the screen while placing a shadowed beautified shape behind the user's sketch. In contrast the sketch shadowing method places the beautified shapes in the foreground and shows user's sketches as shadows.

Beautifying of stylus input into regular geometric shapes requires ongoing accurate recognition; we intend to regulate our recognition algorithm to identify a restricted set of standard geometric elements, such as lines, rectangles, circles, triangles, half-circles and quarter-circles. This set of elements can in turn be combined together to create most shapes. The positioning and size of the shapes is restricted to conform to the grid, which helps to align shapes and aids users in designs where precision is crucial. Users can alter the grid size, thus creating shapes of varying sizes while obtaining accurate measures of the shapes they are creating.

\section{IMPLEMENTATION}

An initial prototype for the Tablet PC has been implemented using C\#.NET. The interface (figure 2) is designed to combat the inherent problems in the Tablet PC environment [7].

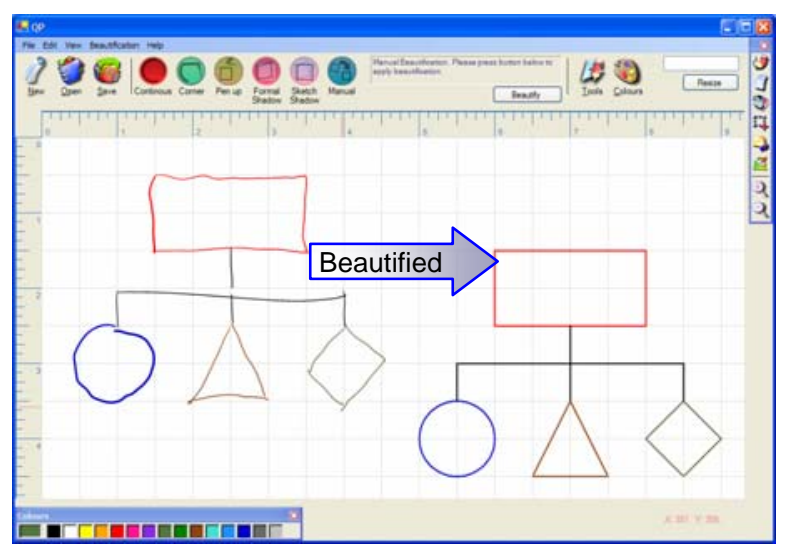

Figure 2. Hand-drawn and beautified renderings of a chart

The raw input strokes are recognised as fragments of a known geometric element contained in our set of shapes using the least square fit algorithm [5]. The beautification techniques differ in implementation from one another and the beautify event is fired at different stages in the drawing process. All beautification techniques require the retention of both the poly-line multifeatured hand-drawn stroke (PMHS) and the poly-line multifeatured beautified stroke (PMBS). Although for continuous, manual, corner and pen-up triggered beautification only the beautified strokes (PMBS) are shown; the raw strokes (PMHS) are held to support accurate beautification. Trials during development suggested that accuracy increased if the shape of a segment is recognised from the raw stroke (before it is snapped to the grid) and, although each segment is recognised individually, by considering the shape of previous adjacent segments better decisions can be made about the current segment. We also found that a direct application of the least-square fit resulted in many straight lines being recognised as curves, particularly on a small grid. We altered the balance of fit to favour straight lines; the balance is dependent on the grid size.

\section{EVALUATION}

Taking a post-task walkthrough approach [8], a pilot survey was used to test the usability of our prototype. The focus of the survey was the accuracy of the recognition and the serviceability of continuous beautification. Participants were asked to draw a fixed series of shapes, after which they answered questions about how they felt while drawing. Response towards the beautification technique was predominantly positive. When asked which shape is the most difficult to draw, most participants identified the small circle. This is due to the small curvature of the four quarter-circles composing the small circle and the recognition sensitivity of the prototype, often classifying curves as straight lines. This has been identified as an area for future fine-tuning.

\section{DISCUSSION AND FUTURE WORK}

One recurring response from the usability study participants was that this is one "cool program"! Even though there is still much refinement to be made to the prototype, it can be ascertained that grid-based design applications with automatic beautification in the tablet-and-pen environment can work. However, more evaluation needs to be carried out to perfect the beautification technique's intuitiveness towards the way we express visual ideas and to examine the effect of the beautification techniques in a variety of design tasks so as to determine the suitability of each.

\section{REFERENCES}

[1] Plimmer, B. and Apperley, M. Interaction with Sketched Interface Designs: An Evaluation Study. Proceedings of SigChi 2004, Vienna, ACM, 2000,. 1337-1340.

[2] Pavlidis, T. and Van Wyk, C. J. An Automatic Beautifier for Drawing and Illustrations. ACM SIGGRAPH Computer Graphics archive, 19(3), 1985, 225-234.

[3] Bolz, D. Some Aspects of the User Interface of a Knowledge Based Beautifier for Drawings. Proceedings of Intelligent User Interfaces '93, 1993, 45-52

[4] Plimmer, B. and Grundy, J. Beautifying Sketch-based Design Tool Content: Issues and Experiences. Proceedings of AUIC Newcastle, 2005, pp. 31-38.

[5] Arvo, J. and Novins, K. Fluid Sketch: Continuous Recognition and Morphing of Simple Hand-Drawn Shapes. Proceedings of UIST '00, San Diego, 2000, 73-80

[6] Igarashi, T., Matsuoka, S., Kawachiya, S. and Tanaka, H. Interactive Beautification: A Technique for Rapid Geometric Design. Proceeding of UIST '97, Banff, 1977, 105-114

[7] O'Brien, L. The Challenge of Designing Interfaces for the Tablet PC. MSDN Resources, http://www.devx.com/TabletPC/Article/21302, accessed 10 January 2005.

[8] Dix, A., Finlay, J., Abowd, G. and Beale, R. Human Computer Interaction, $3^{\text {rd }}$ Edition. Harlow: Prentice Hall, 2003. 